\title{
HETEROPTEROS DE ALICANTE Y ZONAS ADYACENTES
}

\author{
Por J.RIBES ${ }^{1}$ y N.SAULEDA ${ }^{2}$
}

\section{INTRODUCCION}

En este trabajo se recogen observaciones de 312 especies de Heterópteros, repartidos en 193 géneros, de la provincia de Alicante y algunas, numéricamente poco importantes, de las provincias limítrofes de Valencia, Albacete y Murcia . Todo ello es el resultado de una prospección con tendencias preferentes hacia los bioto pos constituídos por arenales y saladares del litoral y del interior. Las referencias a comunidades vegetales vienen expresadas por las normas de clasificación/ de la SIGMA interpretadas con criterios amplios y la lo calización siempre que ha sido posible, se ajusta a las coordenadas UTM. La elección de haber prospectado con mayor intensidad dichos biotopos se justifica por la circunstancia de que estos registros forman parte deun trabajo más amplio, en el que se han estudiado otros grupos de Insectos, las entomocenosis que forman y la relación con los habitats que ocupan.

1. c/Valencia 123-125, ent.3- Barcelona-11.

2. Dpto. Biologia y Geología. Esc.Univ.Form.Profesorado de EGB. Gerona. 


\section{ELENCO DE ESPECIES}

CORIXIDAE Lch.

Corixa panzeri (Fb).- Fondo d'Elx, 30.9.72. Europeo.

Sigara stagnalis (Lch).- Santa Pola, 5.8.73; Laguna del Saladar (Albacete) XH3795, 19.6.75. En lagunas saladas tanto del litoral como del interior. Dos generaciones al año . Euromediterráneo .

Sigara selecta (Fb.).- Salinas de Calp, 21.1.74. Vive en aguas con salinidad débil o mediana. Holomediterrá neo expansivo.

Heliocorisa vermiculata (Pt.).- Santa Pola YH0627, en Arthrocnemum sp., 26.6.74; San Isidro de Albatera, 14. 5.74. Holomediterráneo .

\section{PLEIDAE Fb.}

Plea Leachi MG.\& Kk.- Santa Pola 20.4.74. En aguas encharcadas o de débil corriente, a menudo donde hay un lecho de vegetales importante. Euromediterráneo expan sivo .

\section{NOTONECTIDAE Lch.}

Anisops debilis canariensis Nh. ? - San Isidro de Alba tera XH8926, 14.5.74. Cabo Verde, Madera, Canarias, Sur de Marruecos y Ghana. Se trata de una sola $q$, por lo tanto su determinación debe considerarse con reservas. Notonecta maculata Lch.- Polop YH4780, 16.3.74. Eurome diterráneo .

\section{NAUCORIDAE Fn.}

Naucoris maculatus F.- Santa Pola YH1230, en un azarbe 29.6.73; Polop YH4780, 16.3.74. En aguas estancadas o de débil corriente. Todo el año . Holomediterráneo.

NEPIDAE Lt.

Nepa cinerea L.- En el río Verd YH2060, 20.1.74. Habi- 
tan los charcos poco profundos, fangosos y también en las corrientes. En general pasan el invierno como adultos, hallándose a menudo bajo las piedras cercanas al agua. Viven en aguas de poca salinidad . La puesta suele efectuarse an algas o plantas acuáticas. Los embriones pueden tomar oxígeno por difusión de las -plantas. La raza tipo vive en el norte, centro y par te del sur de Europa . Nepa cinerea poissoni Tam.- Se 1la YH3873, 28.3.75, según el autor se encuentra en el sur de Francia y Cataluña . Nepa cinerea seurati Berg. Realengo XH9130, en Arthrocnemum sp., 14.5.74. Según TAMANINI vive en el sur de Italia, Sicilia, Túnez, Li bia. En la Italia Central hay formas intermedias, in determinables, entre $N$. cinerea seurati y $N$. cinerea ci nerea.

OCHTERIDAE Kk.

Ochterus marginatus Lt.- Sella YH3873, 28.3.75. En las orillas de las corrientes de agua dulce. Salta con facilidad, lo que dificulta su captura. Holomediterrá neo extendido a las regiones etiópica y oriental.

\section{GERRIDAE Lch.}

Gexpis (Aquarius) najas (Deg).- Polop YH4780, 16.3.74. Se mueven con gran agilidad sobre la superficie de las aguas estancadas o con corrientes débiles. Suele ser gregario . Euromediterráneo .

Gerris (Aquarius) cinereus (Fb.).- Relleu YH3473, 17. 6.75. Mediterráneo occidental .

Gerris (Gerris) thoracicus Schml.- Benidorm, 10.11.74. Eurosiberiano .

Gerris (Gerris) gibbifer Schm1.- Polop YH4780, 16.3.74. Euromediterráneo .

Gerris (Gerris) argentatus Schml.- Orihuela XG9796, 1.12.75. En márgenes de lagos y charcas con Amundo do $n a x$. Su medio ambiente tiene usualmente vegetación emergida. Pasa el invierno como adulto. Dos generaciones al año. Euromediterráneo. 
Velia (Plesiovelia) caprai caprai Tam.- Polop YH4780, 16.3.74. Habita en charcas o aguas con corrientes débi les, limpias, con poca materia orgánica. Gregario. Vi ve en lugares con intensidades moderadas de luz . Euro peo.

\section{MESOVELIADAE Dg1, \&. SC .}

Mesovelia vittigera Hv.- San Isidro de Albatera, 17.4. 76. Holomediterráneo extendido a la región etiópica.

HEBRIDAE Fb.

Hebrus dupuisi Wgn.- Sella YH3873, 28.3.75; Cullera YJ3050, en Scirpus sp., 17.4.75. WAGNER det. Mediterrá neo occidental.

HYDROMETRIDAE Bb.

Hydrometra stagnorum (L.).- Sella YH3873, 28.3.75; La Vila Joiosa YH3869, 2.2.75. Eurosiberiano .

MIRIDAE $\mathrm{H}$.

Deraeocoris (Deraeocoris) cordiger (H.).-Petrer XH9364 en Artemisia sp., 8.5.73. Se alimenta de pequeños insectos. Imagos desde mayo hasta agosto . Pasa el invierno como huevo. Especie atlántica.

Deraeocoris (Deraeocoris) ribauti Wgn.- Laguna de Sali nas XH8464, en Suaeda vera, 21.5.74. Vive en Marmbium vulgare. Imagos de mayo a agosto. Pasa el invierno como huevo. Una generación al año. Del sur de Francia Italia y España.

Deraeocoris (Deraeocoris) punctum (Rb.).-Petrer XH 9364 en Artemisia sp., 17.3.73. Imagos de mayo a agosto, se gún WAGNER; dos de nuestras observaciones son del mes de marzo. Pasa el invierno en forma de huevo. Holome diterráneo .

Deraeocoris (Comptobrochis) serenus Dg1.\& Sc.- Petrer XH9364, 30.6.73; Calp BC4581, 8.5.75. En todas partes 
y durante todo el año . Nueva generación en mayo o junio. Holomediterráneo .

Deraecoris (Phaeocapsus) martini (Pt.).- Playa del Saladar YH1735, en vegetación de Ammophizium, 24.5.75.Pa sa el invierno como imago. Se supone que vive sobre Tamarix sp. Holomediterráneo meridional . Nuevo para la Península Ibérica.

Macrolophus caliginosus Wgn.- Petrer XH9365, 14.2.73.WAGNER indica que vive especialmente sobre Inula visco $s a$. Nosotros lo hemos observado además con mucha frecuencia en Ononis natrix, que presenta una viscosidad semejante a la de I.viscosa. El mismo autor señala la presencia de imagos de junio a agosto; nuestras observaciones abarcan todo el año, con un máximo en primavera. Holomediterráneo .

Cyrtopeltis geniculata Fb.- Mazarrón (Murcia) XG5158, en vegetación de Ammophition, 21.3.75, sobre Ononis sp. Holomediterráneo que llega hasta Mongolia .

Dicyphus (Dicyphus) hyalinipennis (Bm.).-Petrer XH9348 9.2.74. De julio a febrero, pasando el invierno, por tanto, en forma de imago . Una generación al año. Holomediterráneo .

Dicyphus (Dicyphus) bolivari Lb.- Campello YH2755, 7. 10.74. Mediterráneo occidental .

Dicyphus (Brachyceroea) albonasutus Wgn.- Petrer XH9364 8.12.73, en Ononis natrix. Imagos de abril a mayo, se gún WAGNER . Holomediterrậneo .

Phitanus maerkeli (H-S.).- Relleu YH3473, 17.6.75. Mírido fitófago, en diferentes plantas de praderas húmedas, especialmente cuando existen juncáceas en ellas. Holártico .

Stenodema (Brachystira) calcaratum (Fn.).- Sant Joan d'Alacant XH9950, en Ammophilion, 14.4.75. Es un insec to que vive en gramíneas en pastos, pantanos y bosques. Euromediterráneo extendido a1 Turkestán .

Notostira elongata (G.).- Pétrola (Albacete), 23.9.74. Es común en lugares con gramíneas, no raro en los márgenes de las carreteras. Tiene relativamente éxito en ambientes alterados. Eurosiberiano .

TrigonotyZus mificornis (G.).- Petrer XH9364, 21.8.73. Especie fitófaga, preferentemente en gramíneas.Adultos 
desde junio hasta octubre. Holártico .

Trigonotylus pulchelzus (H.).- Salobrarejo (Albacete), en Scirpus sp. Insecto fitófago en gramíneas, especial mente Corynephorus sp. Julio y agosto. Pasa el invier no como huevo . Una generación al año . Euromediterrâneo.

Trigonotylus pallidicomis Rt.- Santa Pola YH1129, en Ammophizion, 10.9.73. Imagos en junio y julio según -WAGNER. Pasa el invierno como huevo. Se supone una ge neración al año . Región etiópica, norte de Africa, Ca narias, sur de Francia y Asia Menor. Nuevo para la $\mathrm{Pe}$ nínsula Ibérica'.

Phytocoris (Ribautomiris) minor Kbm.- Petrer XH9364, 25.8.74. Vive en especies de Pinus. Adultos de junio hasta agosto. Pasa el invierno como huevo. Una generación al año. Mediterráneo occidental expansivo .

Phytocoris (Leptophytocoris) virescens Wgn.- Petrer / XH9364, 9.7.73. Según WAGNER vive sobre AnthylZis cyti soides. El único ejemplar que nosotros hemos observado lo fue sobre Ononis natrix . Conocido hasta la fecha sólo de Valencia (tierra típica), Barcelona, Soria y Mallorca.

Phytocoris (Ktenocoris) vittiger Rt.- Laguna de Salinas XH8465, 14.8.73. Forma de vida poco conocida .De junio a octubre. Mediterráneo occidental .

Phytocoris (Eckerlenius) salsolae Pt.- Guardamar YH0520 23.3.74, sobre Suaeda vera. En formaciones de halofitos. Imagos desde julio hasta octubre, según WAGINER ; nuestras observaciones van desde enero hasta junio. Holomediterráneo restringido.

Adelphocoris ticinensis (M.-D.).- Santa Pola YH1231, en Limonietalia, 9.8.73, sobre Limonium sp. Tiene su resi dencia en pantanos, marjales y ciénagas. De junio a septiembre. Pasa el invierno como huevo. Eurosiberia no .

AdeIphocoris Zineolatus (Gz.).- Guardamar YH0722, en Ammophilion, 6.7.73, sobre Ononis natrix . Común. Resi de en diferentes plantas, principalmente en papilionáceas y compuestas. Pasa el invierno como huevo. En USA puede ser plaga de la alfalfa. Holártico.

Calocoris (Calocoris) instabilis Fb.- Redován, 31.1.75. Imagos entre mayo y junio, según WAGNER . Holomedite- 
rráneo discontínuo.

Calocoris (Calocoris) nomegicus (GmI.).-Relleu YH3473 17.6.75. Principalmente en compuestas. Imagos desde ju nio hasta octubre. Pasa el invierno como huevo. Antró pófilo . Holártico.

Calocoris (Closterotomus) trivialis (C.).- $\mathrm{F}^{-1} \mathrm{x}$, 4.72.Holomediterráneo .

Calocoris (Macrocalocoris) nemoralis (F.) ( = hispanus Gm1.).- Benissa, 5.72. Holomediterráneo.

Brachycoleus triangularis (Gz.).- Petrer XH9364, en Eryngium campestre, 29.5.73. Este insecto vive sobre Car dus, Cixsium, de mayo hasta julio. Pasa el invierno como huevo. Una generación al año. Mediterráneo occi dental expansivo.

Taylorilygus palzidulus Bch. (=apicalis Fb.).- Santa Pola YH1733, en Limonietum virgato-furfuracei, 26.10.74. Cosmopolita de zonas cálidas .

Exolygus rugulipennis Pop.- Villena XH8281, 8.9.74. Es pecie ruderal que vive en numerosas plantas, muchas de ellas de cultivo. Pasa el invierno como adulto y es es pecialmente abundante durante el otoño. Dos generació nes a1 año. Holártico. Según KERZHNER, Lygus Hahn -1833, comprende lo que para WAGNER es Exolygus. Lygoco ris Reuter 1875, por lo tanto, y siempre en opinión dē autor ruso, sería el nombre válido frente a Lygus sensU WAGNER .

Exolygus pratensis (L.).-' Santa Pola YH1029, en Suceda vera, 12.10.73. En numerosas plantas . Máximo de pre-sencia en otoño. Holártico.

Exolygus gemellatus (H.-S.).- Els Bassars YH1635, en Arthrocnemon sp., 17.6.74. Según WAGNER vive preferentemente en especies de Artemisia . Dos generaciones. Eurosiberiano.

Exolygus maritimus Wgn.- Laguna de Salinas XH8364, en Suceda vera, 29.8.73, WAGNER det. Diversos autores res tringen sus zonas de residencia al litoral sobre Artemisia, Atriplex, Suaeda vera, Limonium. Nosotros 1o hemos recogido, además de en el litoral, en la laguna de Salinas y Villena, lugares con climas no afectados/ por la proximidad del mar. Prefiere comunidades de ha lófitos. Dos generaciones al año. Nuestras citas van 
desde mayo a noviembre . Se señala que pasa el invierno como imago, aunque no hemos podido recoger ningún a dulto durante esta época del año . Especie atlántica .

Exolygus italicus Wgn.- Santa Pola YH1230, en Suaeda vera, 3.8.73. Todo el año . Mediterráneo occidental .WAGNER det. Los Exolygus citados aquí, exceptuando $E$.mugulipennis y E.pratensis, son muy dificiles de dife renciar y su valor sistemático tendría que ser revisado, 10 que ya señaló R.H. COBBEN refiriéndose a las es pecies de Holanda.

Liocoris tripustulatus (F.).- Monforte del Cid XH9950, en Suaeda vera, 28.9.74. Pasa el invierno como imago. Una generación al año . Eurosiberiano .

Polymemus (Poeciloscytus) cognatus (Fb.).- Sant Joan d'Alacant YH2649, 1.9.73, en Anmophilion, sobre Cakile maritima. Lugares áridos con Salsola, Chenopodium, Atriplex. En el interior hemos recogido esta especie so bre Salsola kali y en el litoral, además, en Launaea resedifolia y Crucianella maritima. Nuestras observaciones van desde mayo a noviembre, con lo que se amplia ligeramente el período indicado por WAGNER, julio-octu bre . Eurosiberiano .

Polymems (Poecizoscytus) unifasciatus (F.).- Playa del Saladar, 21.5.72. WAGNER 1o indica sobre Galium, de ju nio a septiembre. Eurosiberiano.

Capsus ater (L.).- Laguna del Saladar (Albacete) XH3795, 19.6.75. Generalmente en formaciones áridas de grandes gramineas y otras plantas. Pasa el invierno en diapau sa . Holártico.

Capsodes (Horistus) Zineolatus (Br.).-Santa Pola YH1230 1.5.75. Vive en liliáceas, especialmente en Asphodelus fistulosus. Pasa el invierno como huevo. Holomediterráneo expansivo.

Dasyscytus sordidus F.- Petrer, 8.5.73, en Artemisia. Holomediterráneo .

Orthotylus (MeZanotrichus) flavosparsus (C.Sb.).- Monforte del Cid XH9950, Suaeda vera, 28.9.74. Preferente mente en quenopodiáceas. Según WAGNER de junio a ju1io; nuestras observaciones son de septiembre a octubre. Dos generaciones al año. Pasa el invierno como huevo. Holártico . 
Orthotylus (Melanotrichus) schoberiae Rt.- Laguna de Salinas XH8364, en Suaeda vera, 2.6.73. La especie vive, según REUTER, en Schoberia sp. y según JOSIFOV en Suaeda sp. Nosotros la hemos recogido en Suaeda vera. Especie póntica repartida por el sur de Europa.

Orthotylus (Melanotrichas) globiceps Wgn.- Laguna de Salinas, 15.5.73; Santa Pola, 27.5.73; Villena, 17.6.73 Sobre ejemplares de dichas localidades WAGNER describió esta nueva especie para la Ciencia. Uno de nosotros la ha observado posteriormente en Bellpuig (Urgell), Ca taluña.

Orthotylus (MeZanotrichus) moncreaffi Dg1.\& Sc.- Santa Pola, 31.3.73. Vive en Salsola, Arthrocnemum y otros halófitos. Según WAGNER desde fines de julio hasta oc tubre. Pasa el invierno como huevo. Euromediterráneo.

Orthotylus (Melanotrichus) contrarius Wgn.- Salinas, 28.8.68. Descrito de Algeciras, es una especie endémica del sur de la Península.

Orthotylus (Melanotrichus) palustris Rt.- Santa Pola, 8.12.72. Vive sobre Arthrocnerium sp. Imagos de mayo a julio. Pasa el invierno como huevo. Mediterráneo occi dental .

Orthotylus (Melanotrichus) divisus Lv.- Monforte del Cid 28.9.74. Sobre Atriplex halimus . Citado ya de Israel, Libia, Tunicia y España (Murcia).

Laurinia elongata Rbs.- Arenales del Sol, 27.4.75. Un macho es el único ejemplar conocido de esta especie has ta este momento, que encontramos errante en la localidad citada . Descrita por uno de nosotros en "Mediterránea" y comentada posteriormente en la misma Revista .

PiZophorus angustuZus Rt.- Villena XH8281; Vinalopó XH$9551,18.1 .75$. Imagos desde julio a septiembre, según WAGNER . Una de nuestras citas es de enero. Holomedite rráneo .

SysteZZonotus thymi Sgn.- Petrer XH9364, 17.3.73. Vive bajo las plantas en lugares sabulosos y áridos. Holome diterráneo .

Systezzonotus chompioni Rt.- Benidorm, 4.5.68. T.GARCIA leg. Endemismo ibérico, sólo conocido de Soria, Madrid, León, Lérida y Trás-os-Montes . 
Macrotylus (Alzoeonycha) paykulli (Fn.).- Guardamar YH0722, 10.11.73. en el Ammophilion, sobre Ononis natrix. Además lo hemos hallado sobre Inula viscosa. Estas dos especies presentan secreciones viscosas . Otros autores 1o citan sobre 0. spinosa, O.repens, 0.arvensis, en gene ral plantas frecuentes en tierras incultas. Según WAGNER los imagos se encuentran desde mayo a septiembre; nuestras citas cubren todos los meses del año, excepto/ enero y febrero. Se supone que pasa el invierno como huevo. Euromediterráneo .

Macrotylus (Alzoeonycha) atricapilzus Sc.- Campello, 7. 10.74. Según WAGNER vive en Inula viscosa de mayo a julio . Holomediterráneo .

Malacotes mulsanti Rt.- Els Bassars YH1335, 10.4.75. Ina gos en junio-julio y en agosto-septiembre. Probablemen te dos generaciones. En España, norte de Africa y Corfü. Malacotes phlomidis (Lb.).- Petrer XH9465, 27.3.73., en BalZota hispanica. Según WAGNER vive en Phlomis purpurea. Imagos en mayo-junio. Mediterráneo occidental .

Atomoscelis onustus (Fb.).- Monforte del Cid XH9950, en Suceda vera, 28.9.74. Fitófago vive en especies de Atri plex. Imagos desde junio hasta septiembre. Pasa el in vierno como huevo . Holomediterráneo expansivo .

Campy Zonma verbasci(M. -D.).- Monforte del Cid XH9950, en Suceda vera, 28.9.74. En Verbascum sp., pero también en otras plantas. Predator. Dos generaciones al año. Ima gos desde mayo hasta septiembre. Pasa el invierno como huevo . Holártico .

Campy Zomma novaki Wgn.- Playa del Saladar YH1738, en Am mophition, sobre Thymelaea hirsuta, 10.2.74. Uno de nosotros 1o ha capturado asimismo en Llançà (Alt Empordà) Cataluña, sobre la misma planta. WAGNER 10 indica sobre Verbascum nigrum. Imagos desde mayo a junio . Yugoslavia, Italia y Península Ibérica .

Compsidolon (Chamaeliops) crotchi Scott.- Onil XH9976,3.3.75. Imagos desde abril a mayo, según WAGNER; noso-tros tenemos observaciones de febrero y marzo. Pasa el invierno como huevo. Sobre Rosmarinus officinalis . Me. diterráneo occidental .

Compsidolon (Apsinthophylus) balachowskii wgn. (= ribesi Lv.).- Petrer XH9364, 12.1.74. Holomediterráneo meridio na1. 
Compsidolon (Apsinthophylus) sauledai Rbs.- Laguna de Salinas XH8365, en Suaeda vera, 15.5.73; Laguna de Sali nas XH8363, en Suaeda vera, 1.8.73. Hasta la fecha sólo conocido de la Laguna de Salinas.

Pachyxyphus Zineelzus (Ms.\& Rey).- E1 Maigmó, 26.5.72 . Holomediterráneo .

Conostethus venustus (Fb.).- Torrevieja YH0011, 22.2.74. En dunas y playas. Imagos de abril a junio. Holomedite rráneo.

Roudairea Zaunaea Wgn.- Playa del Saladar YH1735, 31.5. 73; Playa del Saladar YH1735, 10.6.73; Playa del Saladar YH1735, 3.6.74; Playa del Saladar YH1735, 17.6.74. Todos ellos en vegetación de Ammophition y sobre Launaea resedifolia, mostrando una gran homocromía con los capí tulos no abiertos de la misma. Todo el material fue re cogido en el curso de este trabajo, no conociéndose nin guna otra localidad de esta especie.

Hadrophyes sulphurelZa Fb. \& Pt.- Santa Pola YH0728, en Arthrocnemum sp., 6.10.73. Común y abundante en las comunidades halofiticas, especialmente en Arthrocnemetalia fruticosae. Segin WAGNER se encuentra en estado de ima go desde mayo hasta julio; nuestras observaciones van desde abril hasta octubre. Holomediterráneo .

Auchenocrepis minutissima (Rb.). - Santa Pola YH0328, en Suaeda vera, 30.9.75. Es común en especies de Tamarix . Imagos desde mayo hasta junio según WAGNER; nuestras ci tas amplian este período hasta septiembre. Pasa el invierno como huevo. Holoméditerráneo.

Tuponia (Tuponia) tamaricis (Pr.).- T. (T.)tamaricis tamaricis (Pr.).- Santa PolaYH0429, en Suaeda vera, 23.5. 74. En especies de Tomarix, dentro de comunidades halofiticas del litoral e interior. Pocas citas, todas ellas de primavera y verano. Holomediterráneo . T. (T.) tamaricis cunealis Rt.- Orihuela XG9898, en Suaeda vera, 3.6. 75. En especies de Tamarix . Mediterráneo occidental .

Tuponia (Tuponia)carayoni Wgn.- Els Bassars YH1536,1.7. 75. En especie's de Tamarix . De mayo a septiembre. Sur de Francia, España y Bulgaria .

Tuponia (ChZorotuponia) brevirostris Rt.- Laguna de Salinas XH8365, 28.6.75. De junio a septiembre. Pasa e1 invierno como huevo . En especies de Tamarix . Holomedi terráneo . 
Tuponia (ChZorotuponia) michalki Wgn.- Santa Pola YH0434 20.4.74. En especies de Tamarix . Según WAGNER de julio a septiembre; nuestras observaciones van desde abril a septiembre. Holomediterráneo .

ANTHOCORIDAE A.\& S.

Anthocoris nemoralis (F.).- Guardamar YH0722, 24.2.72. Euromediterráneo extensivo .

Orius (Orius) niger (W.).- Petrer XH9364, 8.8.73. Es un predator de pequeños animales como trips, arañas rojas, por lo que se encuentra en numerosas plantas. Eurosibe riano .

Orius (Orius) Zaevigatus (Fb.).- Laguna de Salinas XH$8365,15.5 .73$. Holomediterráneo que alcanza las Islas Britânicas .

Orius (Orius) lindbergi Wgn.- Playa del Saladar YH1735, en Ammophizion, 31.5.73, sobre Centaurea seridis . Medi terráneo occidental .

Orius (Heterorius) Zaticollis (Rt.).- Altet, 7.7.72, WAG NER det. Raro . Eurosiberiano .

Lyctocoris compestris (R.).- Petrer XH9364, sobre BaZZO. ta hispanica, 2.11.73. Predator de insectos, chupando también sangre de animales homeotermos. De origen medi terráneo, distribuído por el hombre, es prácticamente cosmopolita.

XyZocoris obliquus (C.).- Cullera (Valencia) YJ3936, en Ammophizion, 17.4.75. Holomediterráneo.

Cardiastethus fasciiventris (Gbg.).- Playa del Saladar YH1738, 2.10.73, sobre ThymeZaea hirsuta. Preda preferentemente sobre áfidos. Subcortical o entre la litera. Todo el año. Mediterráneo occidental que llega a la -Gran Bretaña.

\section{REDUV I IDAE Lt .}

Ploiaria putoni Nh . E1 Saler (Valencia), 11.10.62. De1 sur de Francia y España (Aragón, Cataluña, Valencia y Andalucía).

Ploiaria domestica Scop.- Murcia, canteras, 30.1.77, L. RUIZ leg. Holomediterráneo extensivo . 
Empicoris culiciformis (Deg.).- Requena (Valencia), 23. 9.68. Euromediterráneo extendido al Turkestán y captura do asimismo en Estados Unidos, Argentina y Chile.

Empicoris salinus (Lb.).- Sant Joan d'Alacant YH2649, en Ononis' natrix, en comunidades de Ammophizion, $1.9 .73 ; \mathrm{Pe}$ trer XH9364, en BalZota hispanica, 29.5.73; Laguna de $\overline{1 a}$ Mata YH0412, en Arthrocnemum sp., 21.8.74 . En el suelo bajo halófitos, tanto en el litoral como en el interior. Todo el año. Mediterráneo occidental .

Schidium tibbu (Vs.).- Santa Pola YH0627, en Arthrocnemum sp., 26.6.74; Santa Pola YH0728, en Arthrocnemum sp. 6.10 .73 . Observado en vegetación de halófitos del litoral . En este taxón hay que integrar asimismo los ejemplares catalanes publicados como Metaptems linearis(C.) por uno de nosotros (1961) y posteriormente confirmados por VILLIERS, su autor, que lo describió incluyéndolo en el género Metaptems, aunque previamente WYGODZIMSKY nos indicó, in litteris, que debía encuadrarse en el género Schidium . Conocido sólo del Tibesti, su tierra típica, resulta nuevo, pues, para la fauna europea.

Metapterus Zinearis (C.).- Salobrarejo (Albacete), 16.4. 76. Holomediterráneo .

Polytoxus sanguineus (C.).- Orihuela XG9796, en Amudo donax, 1.3.75. Muy raro . Mediterráneo occidental, nuevo para la Península Ibérica.

Ectomocoris uluZans (R.).- Laguna de Salinas XH8363, en Arthrocnemim sp., 9.10.73; Laguna de Salinas, en Suceda vera, 7.74. Sólo dos observaciones, ambas de los salada res del interior. Holomediterráneo meridional extendido al Asia Central.

Pirates hybridus (Scop.).- Salobrarejo (Albacete),23.9. 74. Mucho más raro que $P$. stridulus. Se señalan como sus preferencias las localidades secas en formaciones esteposas. Holomediterráneo extensivo .

Pirates stridulus (F.).- Altet YH1538, en Arthrocnemum sp., 26.5.74. Parece que muestra en esta Provincia una cierta higrofilia. Todo el año, con máximo en primavera. Mediterráneo occidental.

Cleptocoris strepitans (Rb.).- Santa Pola YH0428, en -Suceda vera, 24.2.74. Insecto errante, se encuentra en ambientes halófilos y en otros que no lo son, tanto en el litoral como en el interior. Todo el año. La f.rufipen 
nis Lc. convive con el tipo y aquí es mâs frecuente que éste. Holomediterráneo extendido a la región etiópica. Vachiria natolica Stål. - Laguna de Salinas XH8365, en Suaeda vera, 14.8.73; Laguna de Salinas XH8464, en Suae da vera, 24.4.73; Laguna de Salinas XH8365, en Suaeda = vera, 9.10.73; Guardamar YH0725, en Suaeda vera, 12.5.73; Alacant YH1848, 26.9.74; Alacant, 1.11.74. Parece que prefiere los ambientes con una cierta halofilia. Se ha citado sobre Tamarix sp. Todo el año . Holomediterráneo meridional.

Coranus niger (Rb.).- Santa Pola YH0328, en Suaeda vera, 2.10.74; Laguna de Salinas YH1635, en Arthrocnemetea, 5. 5.74. Menos frecuente que C.aegyptius, no obstante no se puede considerar raro. Parece que prefiere las comunidades de halófitos. Todo el año. Del Mediterráneo occí dental y Egipto .

Corams aegyptius (F.).- Laguna de Salinas XH8365, en Suaeda vera, 9.10.73. Especie banal que se puede encontrar en gran diversidad de habitats. Todo el año .Holo mediterráneo expansivo .

Rhinocoris erythropus (L.).- Playa del Saladar YH1740, en Ammophizion, sobre Thymelaea hirsuta, 17.8.73. En am bientes muy diversos, se le ha observado predando sobre diferentes animales. Holomediterráneo expansivo.

Rhinocoris cuspidatus Rib.- El Maigmó, 26.5.72. Holomediterráneo .

Sphenadolestes sanguineus (F.).- La Vila Joiosa, 15.4. 72. Mediterráneo occidental.

Reduvius personatus (L.).- Petrer XH9365, 14.4.73.Antro pófilo y cosmopolita.

Reduvius diabolicus Bgv.- Petrer XH9364, 4.6.74. Forma macróptera. Especie muy interesante, que hemos compara do con un ejemplar, igualmente, de la forma macróptera, recogido por ESPAÑ̃L en la Sierra de Cazorla (Jaen) y de terminado por VILLIERS. DISPONS y STICHEL (1959) sólo $1 \frac{1}{0}$ citan de Marruecos y Argelia.

Pasira basiptera Stål .- Elx XH9626, 30.4.74. Forma bra quíptera. Holomediterráneo extendido hasta el Turkestán y Afganistán .

Oncocephalus vicinalis Dsp. (= pilicomis(H.-S.))- Relleu YH3473, 17.6.75. Holomediterráneo expansivo alcan- 
zando el Japón y la región etiópica.

Oncocephazus guzaris Rt.- Alfàs del Pi, 5.5.73.T.GARCIA leg. Muy raro. Endémico de la Península Ibérica. Phymata monstrosa F.- Petrer, 4.76. Holomediterráneo.

\section{NABIDAE C .}

Phorticus velutinus Pt.- Santa Pola YH0328, en Suceda vera, 5.11.74. En el suelo, entre las plantas en lugares húmedos. Sólo lo hemos hallado en el litoral. Holomediterráneo meridional restringido .

Prostemma guttula (F.).- Laguna de Salinas XH8464, en Suaeda vera, 27.3.73. Frecuentemente braquípteros.Se en cuentran en la base de las plantas y arbustos, bajo las piedras. Todo el año. Holomediterráneo expansivo .

Prostemma bicolor Rb.- Elda YH9261, 13.10.74. Mediterrá neo occidental.

Aptus mirmicoides (O.C.).- Polop YH4780; 16.3.74. Euromediterráneo .

HaZonabis occidentalis Kerzh.- Laguna de Salinas XH8364, en Suaeda vera, 10.6.74. Reside y es abundante en las comunidades vegetales halofiticas por nosotros visitadas. Corretea entre los detritus. Mediterráneo occidental.

Nabis capsiformis Gm.- Novelda XH9551, 18.1.75. Cosmopo lita de las regiones cálidas.

Nabis provencalis Rm.- Playa del Saladar YH1738, 10.6.73 en vegetación de Ammophilion, sobre Cakile maritima. In secto que habita en comunidades ruderales. Holomediterráneo de área restringida .

Nabis pseudoferus ibericus Rm. - Santa Pola YH1129, en Ammophilion, 10.9.73. Mediterráneo occidental .

Nabis punctatus C. (=feroides Rm.).-Villena XH7877,26.2. 74. Europeo, con afinidades meridionales.

Nabis reuterianus Pt.- Dénia BD4605, en Ammophilion, 26. 10.73. en Salsola kali . Todo el año . Mediterráneo occi dental.

Aspizaspis viridis viridis Br.- Laguna de Salinas XH8365 en Suaeda vera, 14.11.73; Santa Pola YH0426, en Tamarix sp., 31.3.74. Habita tanto en e1 litoral como en el in- 
terior. Nuestras citas van desde marzo a noviembre. Ho lomediterráneo extendido hasta Mongolia.

\section{LEPTOPODIDAE C.}

Leptopus marmoratus (Gz.).- Orihuela XG9898, en Suaeda vera, 3.6.75. Holomediterráneo expansivo.

Leptopus hispanus Rb.- E1da YH9261, 13.10.74, bajo piedras . Holomediterráneo restringido .

\section{SALDIDAE C.}

Pentacora sphacelata (U.).- Santa Pola YH0929, 27.5.73. En los límites agua-tierra de las lagunas saladas. Ibérico y de las subregiones Neártica y Neotrópica.

HaZosalda concolor(Pt.).- Laguna de la Mata YH0411, 20. 11.73; Santa Pola YH0929, 27.5.73; Calp BC4451, 8.5.75. En los límites agua-tierra de los estanques salados. To do el año . Mediterráneo occidental .

SaZdula saltatoria (L.).- Relleu YH3473, 17.6.75. En los márgenes de las lagunas y aguas con corrientes débiles. Habita tanto en las aguas dulces como en las que poseen una cierta salinidad. Descansa y caza en el barro. Ho lártico.

SalduZa c-album (Fb.).- En el río Guadalest YH5777, 12. 6.75. En lodos, cienos, léganos, fangos. Pasa el invier no como adulto. Holártico .

Saldula palustris (Dg1. \& Sc.).- Santa Pola YH0428, 8.6. 74. Insecto halófilo que habita en el litoral en barros muy húmedos, en el límite agua-tierra de los estanques salados . Euromediterrăneo .

Sázauza setulosa (Pt.).- Playa del Saladar, 15.1.73.SEI DENSTÜCKER det; Salobrarejo (Albacete), 16.4.76. R.H.C믐 BBEN (in STICHEL) da la siguiente distribución: Inglatê rra, Francia, Marruecos y Turkestán. Muy raro . Nuevo para la Península Ibérica.

Saldula palizipes (F.).- Sant Joan d'Alacant YH2551, 22. 5.75. Insecto eurioico en relación con la salinidad. Se supone que prefiere las estaciones del interior . Cosmo polita. 
Hyalochiton komaroffi (Jack.).- Playa del Saladar, 9.72, en Teucrium polium var. maritimum . Holomediterráneo .

Galeatus scrophicus Sd.- Playa del Saladar YH3617, en Anmophilion sobre Launaea resedifolia, 1.7.74; Petrer XH9364, en Launaea resedifolia, 25.8.74. Se indica que reside en Launaea spinosa, aunque nosotros 10 hemos observado siempre sobre Launaea resedifolia, tanto en los arenales del litoral como en los del interior, pero en estos últimos con menor abundancia. Imagos de mayo a oc tubre. Probablemente de origen pontomediterráneo, alcan zando la Península Ibérica por el oeste, las estepas dē Asia central y la India por el este y por el sur el nor te de Africa, Sudán, Senegal, Canarias, islas de Cabo Verde, saltando a Namibia y Transvaal .

Tingis (Tropidocheila) marmibii Val.-Villena, 4.76. en Marrubium vulgare. Holomediterráneo expansivo .

Copium teucrii intermedium (Rey).- Petrer XH9364, 19.6. 74; Playa del Saladar, 6.76 . Siempre sobre Teucrium po lium. Vive en especies de Teucrium. Es de remarcar que en el Ammophilion no lo hemos observado nunca, no obstan te la dedicada prospección sobre T.polium var. maritimum que es relativamente abundante, mientras que lo hemos localizado en pies de T.polium no muy distantes de los pies de la variedad anterior, enraizados donde el substrato inicia el cambio de arenas sueltas a suelo. Medi terráneo occidental . PERICART, que está revisando esté género nos ha indicado, in litteris, que sólo existen tres especies de Copium euromediterráneas: C.clavicome (L.), C.teucrii (Host.) y C.adumbratum (Hv.) esta última erémica. Las razas geográfica y morfológicamente es tán mal delimitadas.

Monosteira ribesi Wgn.- E1x, 12.9.73, sobre el rarísimo y extraordinario Populus euphratica y en Prunus amigdalus colindantes; Busot, 20.9.76, consituyendo plaga en los almendros, Prunus amygdalus. WAGNER menciona a $M$. buccata de Prunus comygdalus, pero en nuestras citas nos parece que se trata de M.ribesi. Holomediterráneo .

Phaenotropis parvula (Sgn.).- Petrer XH9364; Santa Pola YH1129, 10.9.73, en Anmophilion . Siempre en Doryenium suffruticosum . Verano y otoño. Del Mediterráneo occidental y Senega1. 
Agrama atricapiZlum (Spin.).- Santa Pola YH0931, 22.4. 74; Pétrola (Albacete), 19.6.75. Insecto que habita sobre especies de Juncus, Typha. Holomediterráneo expansivo .

\section{PIESMATIDAE A.\& S.}

Piesma quadratum (Fb.) (= rotundicolze Rey).- Els Bas-ssars YH1536, 16.9.74; Pétrola (Albacete), 23.9.74 . En especies de Atriplex. Eurosiberiano .

Piesma kolenatii rotundatum Hv. - Villena XH8281, 8.9.74; Sant Joan d'Alacant YH2551, 22.5.75; Laguna de la Mata YHO412, 9.8.73. Holomediterräneo .

\section{BERYT INIDAE Fb.}

Berytinus montivagus (M.D.).- Petrer, 23.1.73. Euromedi terráneo, conocido también de Canarias.

\section{LYGAE IDAE Sta 1}

Caenocoris nerii (Gm.).- Petrer XH9364, 8.8.73. Siempre 10 hemos observado sobre Nerium oleander. Desde agosto a diciembre. Holomediterráneo extendido a las regiones orienta1 y etiópica.

Graptostethus servus (F.).- Playa del Saladar YH1735, en Ammophizion, 10.1.74; Petrer XH9465, 27.3.73. Lo hemos observado sobre diferentes plantas, especialmente en los arenales. No disponemos de observaciones de verano y sí de las otras tres estaciones. Holomediterráneo difundi do en las regiones etiópica, oriental y australiana.

Lygaeosoma sardeum Spin.- Guardamar YH0722, en AmmophiZion, 10.11.73. En el suelo entre rastrojos. De marzo a noviembre. Holomediterráneo extensivo .

Lygaeus equestris L.- Petrer XH9364, 21.8.73, en Nerium oleander . Eurosiberiano .

Spizostethus furculus (H.-S.) (= festivus Thb).-Alacant YH1848, 26.9.74. Etiópico extendido al área mediterránea meridional.

Spizostethus pandurus (Scop.).- Dénia, 26.10.73. En diversas plantas. Todo el año. Holomediterráneo extendi do por la región oriental. 
Horvathiolus syriacus (Rt.).- Torrevieja, en Thymelaea hirsuta, 22.2.75. Holomediterráneo .

Horvathiolus superbus (Pol1.).- Petrer XH9465, 27.3.73. Muy abundante en los arenales de Petrer, hallándose entre los rastrojos. Holomediterráneo septentrional expan sivo.

Horvathiolus guttatus (Rb.).- Sella YH3873, 28.3.75;Elx XH9626, 30.4.74. Mediterráneo occidental.

Horvathiolus mendosus Hv.- Polop YH4780, 16.3.74. En general raro. Holomediterráneo restringido.

Nysius (Tropinysius) senecionis (Sch1.).- Guardamar YH0722, en Ammophilion, 4.8.73; Laguna de Salinas XH8463, 14.11.73. Común . Todo el año . Holomediterráneo expansivo .

Nysius (Tropinysius) stalianus Hv.- Playa del Saladar YH1735, en Ammophizion, 10.6.73, sobre Launaea resedifo Zia. En formaciones secas. Holomediterráneo.

Nysius (Nysius) thymi (W.).- Elda YH9261, 13.10.74. En lugares secos, en el suelo entre rastrojos . Prefiere los lugares con vegetación dispersa. Holártico.

Nysius (Nysius) ericae (Sch1.).- Alacant YH2148, 26.1.75. En lugares áridos, en el suelo o en numerosas plantas . Holártico . Según SCHMITZ, que lo cita de México, Antillas, Sudáfrica (presumido) y Santa Helena, tiende a con vertirse en cosmopolita.

Nysius (Macroparius) graminicola (K1t.).- Santa Pola YH $1230,3.8 .73$. En rastrojos y hierbas. Todo el año . Ho lomediterráneo expansivo .

Nysius (Macroparius) cymoides (Spin.).- Alacant YH1846, 5.12.74. Holomediterráneo expansivo .

OrthoZomus punctipennis (H.-S.).- Petrer XH9364, en Teu crium polium, 19.6.74. Eurosiberiano.

Cymus meZanocephalus Fb.- Els Bassars YH1536, 9.6.75.En marjales, sobre especies de Juncus, Carex. En el litoral e interior. No lo hemos observado en invierno . Ho lomediterráneo expansivo .

Ischnodemus quadratus Fb.- Salobrarejo (Albacete),28.5. 75. En dunas y marjales con influencia de plantas xerotérmicas . Holomediterráneo expansivo . 
Engistus commendatorius Pt.- Villena XH8077, en Salsola vermiculata, 6.10.74. Nuestras observaciones son de verano y otoño, épocas de1 año que coinciden con las fases de mayor actividad de Salsola vermiculata. Endemismo ibérico.

Engistus boops Df.- Laguna de Salinas XH8464, en Arthro cnemum sp., 27.3.73; Santa Pola YH0727, en Arthrocnemum sp., 20.4.73. En el suelo de las comunidades halofíticas especialmente debajo y entre los restos de Arthrocnemum $\mathrm{sp}$. Tanto en el litoral como en el interior durante todo el año. Holomediterráneo.

Engistus exsanguis Stå1.- Alacant YH1848, 26.9.74. En estepas y eriales. Otoño-invierno. Holomediterráneo de dispersión erémica, nuevo para la Península Ibérica,pero conocido de Mallorca y Canarias.

Henestaris curtulus Hv.-Santa Pola YH1230, en Limonium sp., 3.8.73. Frecuente, en lugares salados del interior y del litoral. Todo el año, con máximo de verano-otoño. Holomediterráneo .

Henestaris Laticeps (Ct.).- Villena XH8077, 6.10.74.Holomediterráneo expansivo .

Henestaris irroratus Hv. - Laguna de Salinas XH8363, en Arthrocnemum sp., 9.10.73; Santa Pola YH0627, en Arthro cnemum sp., 31.3.73. En ambientes salados, tanto del 1i toral como del interior. Mediterráneo occidental .

Geocoris (s.str.) megacephalus (R.).- Playa del Saladar YH1735, en Ammophizion, 31.5.73. Común. Todo el año,con mínimo de invierno . Holomediterráneo expansivo .

Geocoris (s.str.) palzidipennis (C.).-Petrer XH9364,30. 6.73. Raro. Todo el año . Euromediterráneo extendido a la región indoaustral .

Geocoris (s.str.) Zineola (Rb.).- Petrer XH9465, 27.3. 73. Común. Todo el año . con ligero mínimo de invierno. Holomediterráneo .

Geocoris (Piocoris) erythrocephalus (P.\& S.).- Petrer XH9365, 14.2.73. Xerotérmico . Lo hemos observado prefe rentemente sobre Ballota hispanica. Todo el año. Holomediterráneo expansivo .

Stenophthalmicus fajoumensis C.- Playa del Saladar YH1738, en Ammophizion, 18.4.74; Playa del Saladar YH1738 
en Ammophilion, 6.12.73; Els Bassars YH1536, en Limonie talia, 9.6.75; Playa del Saladar YH1738, en Ammophilión 2.10.73. Especie nueva para la Península. Tres citas de dunas arenosas y otra a dos kilómetros de estas formaciones . Especie confirmada por SEIDENSTÜCKER. Citado de Argelia, "Africa occidental francesa", Túnez, Egipto Sudán, Chipre e Israel.

Macroplax fasciata (H.-S.).- Petrer XH9365, 25.4.74, so bre Cistus sp. Holomediterráneo expansivo .

Macroptemella convexa (Fb.).- Santa Pola, 18.8.73. Mediterráneo occidental.

Macropternella conica (Rey).- Gandía (Valencia), en Ammophilion, 15.3.75. Mediterráneo occidental .

Macropternezla marginalis (Fb.).- Petrer, 25.4.74. Medi terráneo occidental .

Microplax plagiata (Fb.).- Santa Pola YH1029, en Limoni um sp., 12.10.73. Holomediterráneo meridional.

Microplax intermuta (Fb.).- Petrer XH9364, en Artemisia sp., 17.3.73. Holomediterráneo .

Oxycarenus Zavaterae (F.).- Guardamar YH0520, en Suaeda vera, 23.3.74. Especie banal . Gregario . En malváceas y otras plantas. Todo el año. Mediterráneo occidental expansivo .

Oxycarenus pallens (H.-S.).- Petrer XH9364, 2.11.73. Ho lomediterráneo .

Artheneis foveolata Spin. - San Fulgencio YH0129, sobre Tamarix sp., 30.4.75. Holomediterráneo .

Heterogaster urticae (F.).- Laguna de Salinas XH8564, 4.6.74. Vive sobre Urtica sp. y también en las raíces de Ammophila amudinacea, esencialmente en habitats cáli dos . Euromediterráneo .

Platyplax inermis (Rb.).- Petrer XH9365, 25.4.74. Holomediterráneo .

Camptocera glaberrima (Wk.).- Laguna de Salinas XH8363, en Arthrocnemum glaucum, 9.10.73. Holomediterráneo de dispersión erémica, extendido a las regiones etiópica y oriental .

Ischnocoris punctulatus flavipes Sen.- Petrer XH9364, en BaZlota hispanica, 2.11.73. Mediterráneo occidental . 
Eremecoris fenestratus (H.-S.).- Salobrarejo (Albacete) 16.4.76. Euromediterráneo expansivo .

Scolopostethus brevis Sd.- Relleu YH3473, 17.6.75. Holo mediterráneo .

Scolopostethus patmulis Hv.- Sierra de Aitana, 19.2.74 T.GARCIA leg. Mediterráneo occidental.

Taphropeltus andrei Pt.- Sierra de Aitana, 19.2.74. T.GARCIA leg. Mediterráneo occidental .

Notochizus crassicomis (Bär).- Calp, 5.9.72. Mediterrá neo occidental. SLATER indica que es sinónimo de $N . f e-$ rrugineus Ms.\& Rey, lo que SERVADEI recoge también en su catálogo, pero vistas las series que poseemos de las has ta ahora consideradas dos especies, nos parece que siguen siendo válidas, reservándonos sin embargo la opinión de finitiva al respecto, hasta que puedan ser estudiadas detenidamente.

Stygnocoris foustus Hv.- E1s Bassars YH1335, en Juncus sp., 22.2.75. Holomediterráneo restringido.

Hyalochilus ovatulus (C.).- Gandía (Valencia), en Ammophilion, 15.3.75. Holomediterráneo.

Paromius Zeptopoides (Bär.).- Gandía (Valencia),15.3.75 Holomediterrâneo .

Remaudierana annulipes (Bär.).- Guardamar YH0520, en Su aeda vera, 23.3.74. Se encuentra abundante durante todo el año en ambientes de salinidad mäs o menos elevada.Ho lomediterráneo extendido a las regiones etiópicas y oriental.

Beosus maritimus (Scop.).- Villena XH7877, 26.2.74. En el suelo bajo diversas plantas. Banal . Observaciones de febrero a octubre. Holomediterráneo expansivo.

Peritrechus gracilicomis Pt.- Santa Pola YH0328, en Su aeda vera, 2.10.74. En hojarasca. Holomediterráneo expansivo.

Peritrechus meridionalis Pt.- Els Bassars YH1335, en Su aeda vera, 22.2.75; Santa Pola YH0728, en Arthrocnemum glaucum, 6.10.73. Holomediterráneo.

Raglius aZboacuminatus (Gz.).- Petrer XH9365, BaZZota hispanica, 14.4.73. Entre hojarasca, rastrojos . Todo el año . Holomediterráneo expansivo. 
Xantochizus saturnius (R.).- Playa del Saladar YH1738,en Ammophilion, 6.12.73. Holomediterráneo .

Lomprodema maurum (F.).- Salinas XH8364, 2.6.73. Todo el año. Holomediterräneo expansivo .

Lasiocoris anomalus (K1t.).- Pétrola (Albacete),23.9.74. Holomediterrâneo .

Lasiocoris antennatus Mtd.- Petrer XH9364, 8.8.73. Ponto mediterráneo, citado ya de Cataluña y Valencia por uno de nosotros.

Aphanus rolandri (L.).- Salinas XH8465, 27.3.75. En for maciones áridas, aunque quizás no tanto como las del sur de la provincia de Alicante. En el suelo entre rastrojos . Euromediterráneo.

Neurocladus brachiidens (Df.).- Salinas, 28.8.68. Holomediterráneo .

Emblethis angustus Mtd.- Alacant YH2148, 17.11.74. Holo mediterráneo extendido al Asia Central .

Emblethis duplicatus Seid.- Guardamar YH0725, en Artemi sia sp., 12.5.73. Holomediterräneo .

Emblethis ciliatus Hv.-Villena XH7977, 6.10.74. Habita en lugares secos, en el suelo entre las plantas. De or $\underline{j}$ gen pontomediterráneo, aparece ya citado del País Valen ciano por uno de nosotros.

Emblethis denticollis Hv.- Cullera (Valencia), en Ammophilion, 17.4.75. Insecto de lugares secos, entre los rastrojos. Holomediterráneo expansivo .

Emblethis griseus (W.).- Calp BC4481, en Arthrocnemum sp. 8.5.75. En formaciones esteparias, en el suelo bajo las plantas. Eurosiberiano. Las citas norteamericanas se refieren a $E$. vicarius $\mathrm{Hv}$.

Emblethis parvus Mtd.- Salinas XH8364, en Suaeda vera,16.9.74; Santa Pola YH0627, en Suaeda vera, 30.3.74. Pa rece que prefiere la vegetación de halófitos. Nuestras observaciones van de febrero a noviembre. Mediterráneo occidental .

Gonianotus marginepunctatus (W.).- Petrer XH9365, 14.2. 73. Insecto de lugares cálidos y arenosos . Reside en el suelo entre la hojarasca. Tenemos observaciones que van desde febrero a noviembre. Eurosiberiano, extendido a la India y región etiópica . Las citas norteameri- 
canas corresponden a Emblethis vicarius $\mathrm{Hv}$.

PYRRHOCORIDAE $\cdot \mathrm{Fb}$.

Pyrrhocoris aptems (L.).- Guardamar, 12.5.73. Muy abun dante en comunidades ruderales, entre hojas de diversas plantas, presentando a menudo un comportamiento gregario. Comen preferentemente semillas, pero a veces pa recen carnívoros y caníbales. No obstante presentar co lores vivos son comidos por pájaros, reptiles. Holár tico.

Scantius aegyptius (L).- Alacant YH1846, 21.1.75.En mu ros, hierbas, piedras... Holomediterráneo extendido has ta el Asia central.

\section{STENOCEPHAL IDAE D1.}

Dicranocephalus setulosus (Fr.).- Playa del Saladar YH1738, en Euphorbia paralias, 2.10.73. Prefiere la cos ta . Todo el año . Holomediterráneo .

Dicranocephalus agilis (Scop.).-Playa del Saladar YH1735, en Ammophilion, sobre Euphorbia paralias,31.5.73.Pasa mucho tiempo en el suelo. En los días cálidos sube a las plantas, corriendo y volando con gran agilidad. Ho lomediterráneo expansivo .

Dicranocephalus marginicollis (Pt.).- Playa del Saladar YH1735, en el Ammophilion, sobre Euphorbia paralias, 2.10 .73 . Prefiere el litoral, hallándose sobre especies de Euphorbia durante todo el año . Mediterráneo occidental.

Dicranocephalus albipes (F.).- Playa del Saladar YH1735, en Ammophizion, 31.5.73, sobre Euphorbia paralias . Ho lomediterráneo expansivo .

\section{COREIDAE Lch.}

Gonocems juniperi triquetricomis (Rb.).-Polop YH4780, 16.3.74. Insecto común en especies de Junipems y otras plantas propias de comunidades no prospectadas . Mediterráneo occidental .

Gonocems insidiator (F.).-Benidorm, 5.9.72. Holomediterráneo . 
Gonocemis acuteangulatus (Gz.).- Benidorm, 5.9.72.Holo mediterráneo expansivo .

Haploprocta sulcicornis (F.).-Torrevịeja, 22.2.74.Pare ce que prefiere las especies de Rumex. Holomediterráneo expansivo .

Enoplops ventralis D.- Salịnas XH8564, 4.6.74. Mediterráneo occidental.

Centrocoris variegatus K1t.- Alacant YH2148, 17.11.74. Nuestras observaciones parecen indicar una preferencia por los ambientes con vegetación nitrófila; no obstante parece que se encuentra aquí y allá. Holomediterrá neo expansivo .

Spathocera Zobata (H.-S.).- Villena XH8281, 8.9.74. Ho lomediterráneo extendido al Asia central .

Prionotyzus brevicornis (Ms. \& Rey).- Villena, 14.4.76. Holomediterráneo .

PhyZZomorpha Zaciniata (Vi1.).-Villena. XH8281, 6.11.73. Especie banal. En y bajo diversas plantas. Holomedite rráneo .

Arenocoris intermedius (Jak.).-Petrer XH19364, 2.11.73; Playa del Saladar YH1735, en Ammophilion, 3.6.74. Tanto en los arenales del litoral como en los del interior. Nuestras citas van desde marzo hasta noviembre. Holomediterráneo .

Arenocoris waltli (H.-S.).- Villena XH7877, 26.2.74.Pre fiere los habitats arenosos y secos con vegetación dis persa. Pasa el invierno como adulto y se supone que tie ne una generación al año . Holomediterráneo expansivo.

Loxocnemis dentator (F.).-Petrer XH9364, 30.6.73. En $\underline{O}$ nonis natrix y otras papilionáceas . Holomediterráneo. Bothrostethus annulipes (C.).- Guardamar YH0621, 23.3. 74. Una observación de los arenales del litoral y otra de los del interior, Petrer 25.4.74. Holomediterráneo expansivo .

Anoplocems sp.-Almansa (Albacete), 5.72. Una of en muy ma1 estado, indeterminable .

Coriomeris affinis (H-S.).-Villena XH8282, 6.11.73. Ho lomediterráneo expansivo .

Coriomeris hirticomis (F.).-Sant Joan d'Alacant, YH2550, 
en Anmophilion, 14.4.75. Holomediterráneo expansivo .

Strobizotoma typhaecomis (F.).- Pétrola (Albacete), 16. 4.76. Holomediterráneo expansivo.

ALYDIDAE D1.

Micrelytra fossuzarum (R.).- Polop YH4780, 16.3.74. Ho lomediterráneo .

Alydus calcaratus (L.).- Santa Pola YH0528, en Arthroc nemum glaucum, 17.8.74. Las larvas son mirmecomorfas, habiendo sido halladas en hormigueros de Formica rufa, Lasius niger,... Se puede alimentar de larvas de hormigas o de semillas. Usualmente de julio a septiembre. Holártico .

Camptopus Zateralis (Gm.).- Villena XH8281, 8.9.74. Ho lomediterráneo expansivo .

RHOPALIDAE A.\& S.

Corizus hyoscyami (L.).- Sant Joan d'Alacant YH2649, 22. 11.73. Fundamentalmente en localidades áridas y soleadas de costas arenosas. Muchos lo asocian a especies de Ononis, pero es polífago. Es un insecto tardio que pasa el invierno como adulto . Eurosiberiano.

Lyorhyssus hyalinus (F.).- Playa del Saladar YH1735, en el Ammophizion, 31.5.73. Todo el año, con máximo de ve rano-otoño . Cosmopolita de las regiones cálidas y tem pladas.

Brachycarenus tigrinus (Sch1.).- Villena XH7877,26.2.74 En suelos áridos. Eurosiberiano.

Stictopleums synavei Göllner-Sheiding.- Santa Pola YH0627, 26.6.74. Endémico ibérico.

Stictopleurus punctatonervosus G1.- Alacant YH2148, 26. 1.75. Eurosiberiano, extendido al sur de China y Nepal. StictopZeums abutizon (R.).- Playa del Saladar,10.6.73. Eurosiberiano .

Maccevethus errans F.- Alacant YH1546, en Ammophizion, 22.10.74. Mediterráneo occidental .

Maccevethus caucasicus Klt.- Villena XH7977, 6.10.74 Ho lomediterráneo . 
Agraphopus lethierryi Stål .- Salinas XH8564, 4.6.74.Holomediterráneo extendido por el Asia Central .

Chrosoma schillingi (Sch1.).- Villena XH8281, 9.9.74 ; Salinas XH8465, 14.8.73 . En formaciones áridas de du nas o lugares incultivados, se pasea despacio por las plantas y su figura y color le dan semejanza con el en torno . Euromediterráneo expansivo .

\section{SCUTELLERIDAE Lch.}

odontoscelis fuliginosus (L.).- Playa del Saladar, YH 1738, en Ammophilion, en Lotus creticus, 2.10.73. Son variables de color. Fuertes minadores. Euromediterra neo, extencido hasta el Turkestán .

odontoscelis lineola Rb.- La Mata YH0610, en AmmophiIion, bajo Lotus creticus, 20.11.73; Playa del Saladar YH1738, en Ammophilion, bajo Crucianella maritima,9.13. 74. Sólo hallado en el litoral, en ambientes arenosos dentro del Ammophilion. Todo el año, con un máximo de otoño . Conocido de Alemania, Suiza, Francia y Cataluña .

Irochrotus sp.- Villena XH7977, 6.10.74 . Restos proba blemente de I.maculiventris $\mathrm{Gm}$. Elemento holomediterrá neo meridional.

Solenosthedium bilunatum (Lef.).- Sant Joan d'Alacant YH2649, 22.11.73; Salinas XH8463, 14.11.73. Holomedite rráneo meridional .

Odontotarsus purpureolineatus (R.).- Playa del Saladar YH1735, en Ammophilion, 10.1.74. Holomediterráneo expansivo .

Odontotarsus caudatus (Bm.).- Alacant, 16.4.72. Holomediterráneo .

Eurygaster austriaca seabrai Ch.- Alcoi, 25.5.72; Ala cant, 5.72; Gata, 14.5.72. Endemismo ibérico.

Eurygaster testudinaria (G.).- Salobrarejo (Albacete), 23.9.74. Prefiere ambientes húmedos donde la hierba es alta. Vive en Juncus, Carex, . Una sola generación al año . Eurosiberiano . 
Ventocoris falcatus (Cyr.).- Sant Vicent del Raspeig, 14.4.72. Holomediterráneo .

Tarisa flavescens A.\& S.- Salinas XH8564, 28.6.75; Ala cant YH1546, 22.10.74. Nosotros 1o hemos observado siem pre sobre Salsola vermiculata. Se cita como insecto que habita en especies de Artemisia. Holomediterráneo meridional.

Tarisa hispanica Wgn.- Santa Pola YH0328, 2.10.74;Sali nas XH8464, 21.5.74; Santa Pola YH0528, 23.8.74. En el litoral e interior sobre Beta sp. Endemismo ibérico, STICHEL lo cita de Argelia .

Ancyrosoma Zeucogrammes (G1.).- Petrer XH9364, 4.6.74. Holomediterráneo expansivo .

Graphosoma semipunctatum (F).- Playa del Saladar XH1738, en Ammophilion, sobre Eryngium maritimum, 2.10.73; Petrer XH9364, en Eryngium compestre, 30.6.73. Común en el litoral e interior, en las plantas citadas. Verano otoño. Holomediterráneo .

Graphosoma Zineatum italicum (M1.).- Benidorm, 2.5.72. Euromediterráneo extensivo .

Menaccamus arenicola (Sch.).- Petrer XH9365, 14.2.73 ; Playa del Saladar XH1738, en Ammophilion, 24.3.73. En formaciones arenosas, en las raíces de las plantas.Pre senta adaptaciones profundas a la vida en las arenas. En el litoral e interior, durante todo el año. Holome diterráneo.

Sciocoris fissus Ms. \& Rey.- Salinas XH8365, en Suaeda vera, 15.5.73; Santa Pola YH0328, en Suaeda vera, 30.9. 75. Es común en los saladares, tanto del litoral como del interior. Todo el año . Con máximo de verano y mí nimo de invierno. Mediterráneo occidental.

Sciocoris (Neosciocoris) conspurcatus majusculus Lv.

(Sciocoris conspurcatus majusculus) Linnavuori,1964. Ann.Zoo1.Fenn., 1:308 (Le Lavandou).

(Sciocoris (Neosciocoris) carayoni) Wagner, 1965. Ac ta ent. Mus. Nat. Pragae, 36: 136-138 (Banyuls, Sts. Maries-de-1a-Mer, Hyères, Fréjus, Barcelona,E1 Prat de Llobregat, Castelldefels, Albufera de Valencia, Perellonet). Nov.syn . 
Playa del Saladar YH3617, en Ammophition, 1.7.74. Vive en el litoral sobre substratos arenosos, durante todo el año, con un mínimo de invierno . Mediterräneo occidental .

Sciocoris helferi Fb.- Laguna de Salinas XH8465, 27.3. 75; Santa Pola YH1129, 10.9.73. En el suelo bajo los de tritus de las plantas. Holomediterráneo extendido has ta el Asia Central.

Aelia acuminata (L.).- Salinas XH8465, 27.3.75. En diversas comunidades vegetales. En algunos lugares y años puede ser plaga menor del trigo . Eurosiberiano. Aelia cognata Fb.- Villena XH8281, 8.9.74. Holomediterrâneo.

Aelia germari Ks.- Monforte del Cid, 18.3.72. Holomedi terráneo .

Neottiglossa Zeporina (H.-S.).- Salobrarejo (Albacete), 23.9.74. Holomediterrăneo expansivo .

Eysarcoris inconspicuus (H.-S.).- Guardamar YH0722, 4. 8.73. Entre y sobre numerosas plantas, durante todo e1 año, con un máximo de primavera-verano. Holomediterráneo alcanzando el Asia Central y zonas adyacentes de las regiones oriental y etiópica.

Holcostethus strictus (F.).- Alacant YH1848, 26.9.74. Holomediterráneo .

Holcostethis sphacelatus (F.).- Salinas XH8464, 14.11.73. Holomediterráneo expansivó.

Croantha ornatula (H.S.).- Alacant YH1848, 26.9.74. En comunidades halofíticas o ricas en nitrógeno, presenta un máximo de frecuencia en el otoño. Holomediterráneo meridional, que alcanza el Asia Central .

Carpocoris mediterraneus atlanticus Tam.- Santa Pola YH1230, 29.6.73. Aquí y allá, durante todo el año, con un máximo en verano-otoño . Mediterráneo occidental .

Codophila vaxia (F.).- Alacant, 10.11.74. Insecto muy variable, que habita en diferentes plantas de múltiples comunidades vegetales. Todo el año. Holomediterráneo expansivo .

Antheminia absinthi (Wgn.).- Villena XH8281, en Artemi sia sp., 6.11.73. Sur de Francia, Portugal y España . 
Dolycoris baccarum (L.).- Petrer XH9364, 19.6.74. Vulgar. Vive sobre numerosas plantas comiendo flores,fru tos, semillas y parece que también áfidos y huevos de coleópteros. Durante el cortejo nupcial se presentan agregaciones . Eurosiberiano extendido a la región oriental.

Brachynema cinctum (F.).- Alacant YH2148, 26.1.75. Pa rece preferir comunidades halofíticas. Holomediterráneo.

Brachynema purpureomarginatum triguttatum $\mathrm{Fb}$.

(Brachynema triguttata) Fieber, 1870, Verh.Ges.Wien, 20: 263 (Andalucía).

(Brachynema espagnoli) Ribes, 1965. Mis. Zool.,2(1)

59-61 (Nova Tabarca, Totana). Nov. syn .

Salinas XH8364, en Suaeda vera, 24.2.73; Santa Pola YH 1330, en Suaeda vera, 15.1.74. Común, especialmente en comunidades halofiticas. Se encuentra durante todo el año, con un mínimo en invierno. Elemento del SE ibéri co . Uno de nosotros está trabajando ahora en la revisión del género Brachynema Ms.\& Rey . Entre los diferen tes tipos estudiados se ha visto que el de Cimex purpu reomarginatus Rambur, 1842, prestado amablemente por el Dr. W.R.Dolling, del British Museum, de Londres, no es reierible en absoluto a $B$. cinctum (F.)., como han in dicado todos los autores hasta la fecha; por el contra rio hay que asimilarlo a las formas del grupo trigutta tum $\mathrm{Fb}$.-hypocrita Pt. (con la base del escudete trimaculada), cuyo status taxonómico será objeto de discusión en su momento. La sinonimia expuesta aquí no es, por consiguiente, más que un adelanto de lo que se pu blicará al término del estudio actualmente en curso.

Brachynema germari (K1t.).- Santa Pola YH1231, en Suae da vera, 9.8.73; Salinas XH8363, en Suaeda vera, 1.8.7 $\overline{3}$. Holomediterráneo extendido al Asia Central .

Holoogaster fibulata (Gm.).- Oril XH9976, 17.11.73. Ho lomediterráneo .

Eurydema ventraZe Klt.- Alacant YH1848, 20.10.74.Común durante todo el año . Holomediterráneo expansivo .

Eurydema omatum (L.).- Re1leu YH3473, 17.6.75. Eurosi beriano extendido por la región oriental.

Eurydema oleraceum (L.).- Elda YH9261, 13.10.74.Común. Eurosiberiano . 
Acrosternum millieri (Ms. \& Rey).- Monforte del Cid XH 9950, 28.9.74. Holomediterráneo meridional expansivo .

Nezara viridula (L.).- Calp BC4581, 8.5.75. En diversas plantas durante todo el año . Cosmopolita de las zonas câlidas .

Piezodoms lituratus (F.).- Petrer XH9364, 25.8.74. En numerosas plantas . Euromediterráneo expansivo .

Rhaphigaster nebulosa (Pd.).- Santa Pola, 17.8.74. En diferentes plantas. Holomediterráneo expansivo .

Zicrona caemulea (L.).- Villena XIH7877, 26.2.74. Depre dador . Holártico extendido a la región oriental.

\section{CYDNIDAE Bb.}

Canthophomis meZanoptemis meZanoptemis (H.S.).- Playa deI Saladar YH1638, 24.3.73. En numerosas comunidades y plantas . Especialmente en primavera . Holomediterrá neo .

Crocistethus waltzianus (Fb.).- Petrer XH9365, 14.2.73; Playa del Saladar YH1735, 10.1.74. En arenales del interior y del litoral. Holomediterráneo.

Ochetostethas tarsalis Ms.\& Rey.- Playa del Saladar YH 1736, 1.7.74; Villena XH8281, 8.9.74. En las raíces de numerosas plantas, durante todo el año . Material estu diado por KERZHNER . Mediterráneo occidental .

Cephalocteus scarabaeoides (F.).- Mar Menor (Murcia)en Amnophilion, 26.1.74; Mar Menor, en Ammophilion, 28.8. 74. Enterrado al pie de numerosas plantas en lugares a renosos, especialmente del litoral. No lo hemos halla do en la provincia de Alicante; parece, por tanto, que el Mar Menor sería su limite norte en nuestra Península . Holomediterráneo restringido.

Byrsinus albipennis (C.).- Playa del Saladar YH1735, en Ammophizion, 10.1.74; Playa del Saladar YH1738, en Ammophilion, 6.12.73. Vive en las raices de las plantas en los arenales del litoral; no lo hemos hallado enlos arenales del interior. WAGNER encuentra este insecto relacionado con Aethus pilosulus, Macroscytus brunneus y Cydnus aterrimus, todos ellos bajo Ammophiza arenaria. Mediterráneo occidental.

Aethas flavicornis (F.).- Petrer XH9365, 14.2.73. Adap 
tado a la vida en el suelo, se encuentra en las raíces de diversas plantas. Prefiere los substratos arenosos del interior. Holomediterráneo expansivo .

Aethus Zaticolzis Wgn.

(Aethus Zaticoliis) Wagner, 1954. Comm.Biol.,14 (2):

1-3 (Jable, Maspalomas, Galdar, Graciosa, Cartagena). (Aethus perisi) J.A. de la Fuente, 1970. EOS, 45 : 162-163 (Alicante). Nov. syn.

Laguna de Salinas XH8464, en Suaeda vera, 27.3.73; Santa Pola YH0627, en Sucueda vera, 30.3.74. Especie marcadamente halofila, vive enterrada al pie de especies de Arthrocnemum y Suaeda en comunidades de ArthrocnemetaIia fruticosae, tanto en el litoral como en el interior, durante todo el año, con un máximo en primavera-verano. Ibérico meridional y canario.

Aethus pilosulus (H.-S.).- Guardamar YH0521, en Sudeda vera, 22.2.74. En el suelo en lugares con salinidad no table. Holomediterráneo .

Aethus pizosus (H.-S.).- Salinas, 27.3.73; Río Segura YH0519, 1.6.75. Holomediterráneo .

Macroscytus brunneus (F.).- Petrer XH9364, 19.6.74. En terrado bajo diferentes plantas, 10 hemos observado muy abund ante bajo Thymelaea hirsuta en las arenas del 1itoral, donde halla un microclima menos extremo que el de las arenas desnudas. Holomediterräneo extendido por las regiones etiópica y oriental.

Geotomus Zongicomis Wgn.- Realengo XH9132, en Suaeda vera, 30.1.74. Endémico de la Península.

Geotomus elongatus (H.-S.).- Sierra de Aitana, 12.2.74; Moraira, 22.7.72. Holomediterráneo expansivo .

Geotomus petiti Wgn.- P1aya de1 Saladar YH1835, 27.4.75. Mediterráneo occidental.

Geotomus punctulatus (C.).- Calp BC4581, en Ammoptition 22.1.74. Holomediterráneo expansivo .

Cudnus aterrimus (Fst.).- Petrer XH9364, 19.6.74.Común en ambientes más o menos humanizados. Todo el año, ex cepto en invierno. Especie de amplia dispersión, se en cuentra en las regiones paleártica, etiópica e indoaus tra1.

Thyraeocoris fulvinervis (Sc.).- Elx, 13.5.72. Holomediterráneo . 


\section{SUMMARY}

Data are recorded in the present communication concerning three-hundred and twelve species of Heterop tera from the SE of the Iberian Peninsula, including seven species new to this area: Deraeocoris (Phaeocapsus) martini (Pt.), Trigonotylus pallidiconnis Rt.,Schi dium tibbu (Vs.), Polytoxus sanguineus (C.), Saldula sē tulosa (Pt.), Engistus exsanguis Stå1 and Stenophthalmicus fajoumensis $\mathrm{C}$., one of them being reported for the first time in Europa, Schidium tibbu (Vs.). At the species-level, the following changes are proposed:Scio coris (Neosciocoris) conspurcatus majusculus Lv. $=$ Scio coris (Neosciocoris) carayoni Wgn., nov. syn., Brachyne ma purpureomarginatum triguttatum $\mathrm{Fb}$. = Brachynema $\overline{\underline{s}}$ pagnoli Rbs., nov. syn. and Aethus laticollis Wgn. ” Aethus perisi J.A. de la Fuente, nov. syn. 


\section{BIBLI OGRAF-I A}

COBBEN, R.H., 1958.- Biotaxonomische Einzelheiten über niederlan dische Wanzen (Hem.,Het.), Tijd.v.Ent. 101 (1):1-46.

COULIANUS, C-C \& OSSIANILSSON, F., 1976.- Catalogus insectorum sue

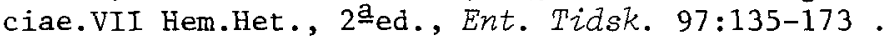

DANILOVITSH,L.P., 1975.- Distribución y variabilidad geográfica de odontoscelis fuliginosa L. y O.byrrhus Seid. (Het.Scutelleridae) (en ruso). Nasekomye Mongolii. 3:86-89.

DRAKE, C.J. \& RUHOFF, F.A., 1965.- Lacebugs of the World. A Ca talog, Smiths. Inst., 243:1-634.

FIEBER, F. X., 1860-61.- Die europäischen Hemiptera, Wien.1-444.

FUENTE, J.A. de 1a, 1969. - Las especies españolas del género Ae thus Dall. EOS 46:85-107.

FUENTE, J.A. de la, 1972.- Revisión de los Pentatómidos ibéricos. Fanilia Cydnidae Billberg, 1820. Bol.R. Soc.Española Hist. Nat. (Biol.) 70:33-78.

GRAVESTEIN, W.H., 1969.- Twaalf nieuwe Heteroptera voor de fauna van Mallorca . Ent.Ber. 29:156-158.

GRAVESTEIN, W.H., 1978.- Hemiptera Heteroptera new to the Baleares, in particular to the Island of Mallorca .Ent.Ber.38:37-39

GÖLLNER-SCHEIDING, V., 1975.- Revision der Gattung Stictopleurus Stål 1872 . (Het. Rhopalidae), D. ent.Zeit, N.F. 22:1-60.

HOBERLANDT, L., 1955.- Results of the zoological scientific expe dition of the National Museum in Praha to Türkey .18 Hemiptera IV. Terrestrial Hemiptera-Heteroptera. Sup1.3: 1-264.

JoSIFOV, M.,1960/64/69.- Artenzusammensetzungen und Verbreitung der Insekten von der ordnung Heteroptera in Bulgarien (en búl

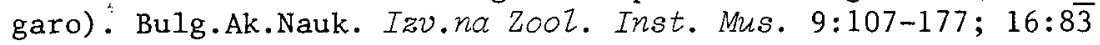
150; 29:29-82.

JOSIFOV, M., 1965.- Zur Systematik der Gattung Melanocoryphus Stal (Hem. Het. Lygaeidae). Acta ent.Mus. Nat. Pragae. 36:311-344

JOSIFOV, M., 1966.- Zur Systematik der Gattung Maccevethas Dallas 1852 (Hem.Het. Rhopalidae). Reichenbachia 8(7): 55-61.

KERZHNER, I.M., 1963.- Beitrag zur Kerntnis der Unterfamilie Na binae (Het. Nabidae). Acta ent.Mus. Nat. Pragae. 35:4-61.

KERZHNER, I.M., 1976.- Heterópteros nuevos y poco conicidos de Mongolia y regiones adyacentes de la URSS (en ruso). Nasekomje Mongolii . 4:30-86.

LANDSBURY, I., 1965-66.- A revision of the Stenocephazidae Dallas 1852. (Hem.Het.) Ent.Mont.Mag. 101:52-92/145-160. 
LINDBERG, H., 1932.- Inventa entomologica itineris Hispanici et Maroccani, quod a. 1926 fecerun Harald et Håkan Lindberg, XIII, Hem.Het. (exc1. Capsidae et Hydrobiotica) .Comm. Biol. 3 (19): $1-53$.

LINNAVUORI, R., 1964.- Hemiptera of Egypt, with remarks on some species of the adjacent Eremian region. Ann.Zool.Fenn.1:306355 .

LINNAVUORI, R., 1972.- Studies on Palaearctic Hemiptera .Ann.Ent. Fenn. 38 (1):40-50.

NIESER, N., in J.ILLIES, 1978.- Limnofauna europaea, Gustav Fischer Verlag. Stuttgart. New York, Swets \& Zeitlinger B.V.Amsterdam, 2a ed.: 280-285.

PÉRICART, J., 1972.-Hémiptères Anthocoridae, Cimicidae et Micro physidae de Z'ouest-paZéarctique. Masson et Cie. 7:1-402.

PÊRICART, J., 1975.- Introduction à une revision des Piesma paléarctiques. Ann. Soc. Ent. Fr. (N.S.) 11(3):517-540.

POISSON, R., 1957.-Héteroptères aquatiques. Faune de France . Pa ris. 61:1-263.

PRIESNER, H. \& ALFIERI, A., 1953.- A Review of the Hemiptera Heteroptera known to us from Egypt. Bulz. Soc. Fouad 1 $1^{2}$ d'Ent. $37: 1-119$.

PUTSHKOV, V.G., 1961-1969.- Fauna de Ucrania (en ucraniano). Ak. Nauk. Ukr.RSR./ Inst. Zool.21(1):1-338; (2):1-162; (3):1-388.

RIBES, J., 1961.- I Contribución al estudio de los Reduviidae de Cataluña . Mis. Zool. 1(4):57-73.

RIBES, J., 1973.- Sobre Empicoris salinus (Lidberg), 1932. Mis. ZooZ. 3 (3):33-42.

RIBES, J., 1976.- Dos Míridos nuevos de la provincia de Alicante. Mediterránea, 1:33-46.

RIBES, J., 1977.- Un Mírido nuevo y otro ya conocido del País Va lenciano. Mediterránea 2:29-34.

SCHMITZ, G., 1976.- La faune terrestre de 1'île de Sainte-Hêlène 20 Heteroptera, 5 Lygaeidae. Mus. Roy.Afr. Centr. Ann.Sc.Zool. 215:391-410.

SEABRA, A.F., 1924-1934.- Sinopse dos Hemípteros Heterópteros de Portuga1 . Mem. Est. Mus.Zool. Univ. Coimbra Ser.1,1 (1-16): 1-517

SEIDENSTÜCKER, G., 1967.- Untersuchungen an Emblethis (Het.Lygaei dae). Reichenbachia 8: 249-266.

SEIDENSTÜCKER, G., 1972.- Eine neue Form aus Odontoscelis Lap.aus Türkei (Het.Scutelleridae) .Mitt.Mirch.Ent.Ges. 61:107-113.

SERVADEI, A., 1967.- Rhynchota. Catálogo topográfico e sinonímico. Fauna d'Italia . 9:1-851.

SLATER, J.A., 1964.- A Catalogue of the Lygaeidae of the Wortd. Univ.Connecticut . Storrs: 1-1668. 
SOUTHWOOD, T.R.E. \& LESTON, D., 1959.- Land and water bugs of the British Isles. F.Warne \& Co. Ltd. 1-436.

TAMANINI, L., 1973. - Priorità e sinonimia de Nepa cinerea L. e N. rubra L., (Hem.Het. Nepidae).St.Trent. Sc.Nat. B. 50 (2): 222-259.

TAMANINI, L., 1973.- Studio sistematico e corologico degli Emi tteri eterotteri delle isole Egadi, Eolie e di Ustica .Bolz. Sed.Acc.Gionia Sc.Nat. Cat. 11 (9-10):9-88.

VIDAL, J., 1949.- Hémiptères de I'Afrique du Nord et des pays circum-mediterranées .Mém. Soc. Sc.Nat. Maroc. 48:1-235.

VILLIERS, A., 1960.- Révision des Réduviidés africains . III.Reduviinae. Genre Pasira stål. BulZ. I.F.A.N.29 (A,3):1026 1038 .

VILLIERS, A., 1960.- Hêmiptères Réduviidés récoltés du Tibesti par Ph. Bruneau de Miré . BuZZ. I.F.A.N.22 (a,5):1315-1330.

WAGNER, E., 1961.- Beitrag zur Systematik der Gattung Monostira Costa 1860 (Hem.Het.Tingidae). Mis.Zool. 1(4):75-86.

WAGNER, E., 1965.- Die taxonomische Bedeutung des Baues der Ge nitalien des Männchens bei der Gattung Sciocoris Fallén, $182 \overline{9}$ (Hem.Het.Pentat.) Acta Ent.Mus.Nat. Pragae 36:91-167.

WAGNER, E., 1970-1975.- Die Mixidae Hahn, 1831, des Mittelmeerraumes und der Makaroneschen Inselm (Hemiptera, Heteroptera), 1,2,3.-3 Vols. Ent. Abhandl. 37(Sup1.):1-484; 39 (Supl.) $1-421 ; 40$ (Sup1.) :1-483.

WAGNER, E., 1976.- Vier neue Miriden (Het. Miridae) aus den westlichen Mittelmeerraum . Not.Entom. 61:10-14.

WAGNEF, E., 1976.- Was ist Compsidolon (Apsinthophyzus) ribesi Linn.? (Hem.Het.Miridae). Reichenbachia 16 (12):147-149.

WAGNER, E. \& WEBER, H.H., 1964.- Hétéropteres Miridae.Faune de France. Paris 67:1-591.

WYGODZINSKY, P.W., 1966.- A monograph of the Emesinae .BulZ.Am. Mus. Nât. Hist. 133:1-614.

PIC, M., 1952.- Nouveaux coléoptères du Maroc . C.R. Soc.Sc.Nat. et Phys. Maroc, 3: 65 .

PUEL, L., 1907.- De la variabilité de coloration et des moeurs du Xyletinus (Trachelobrachys) sanguineocinctus Fairm. I'Echange, 23: 146-147.

REITTER, E., 1901.- Byrrhidae (Anobiidae) und Cioidae . Best.Tab. eur. Col., 47:28-33. 